\title{
Factors Affecting Ethanol-Induced Conditioned Place Preference and Locomotor Sensitization in Mice
}

\author{
Chikako Shimizu, ${ }^{*}, a$ Yasuhiro Oki,${ }^{a}$ Yutaka Mitani, ${ }^{a}$ Takeshi Nakamura,${ }^{a}$ and \\ Toshitaka Nabeshima ${ }^{b, c}$ \\ ${ }^{a}$ Frontier Laboratories of Value Creation, SAPPORO BREWERIES LTD.; Yaizu, Shizuoka 425-0013, Japan: \\ ${ }^{b}$ Nabeshima Laboratory, Meijo University; Nagoya 468-8503, Japan: and ${ }^{c}$ NPO Japanese Drug Organization of \\ Appropriate Use and Research; Nagoya 468-8503, Japan. \\ Received August 12, 2015; accepted September 8, 2015; advance publication released online September 16, 2015
}

The rewarding effects of alcohol can lead to progressively heavier and more frequent drinking. Since studies of reward have mainly focused on responses to higher alcohol doses, the relations between reward and moderate/sustained alcohol exposure remain unknown. Our objective was to evaluate factors affecting the reward value of low alcohol doses and risk factors for increasing alcohol doses due to reward progression caused by alcohol exposure patterns. We thus performed conditioned place preference (CPP) and ethanol (EtOH)-induced locomotor sensitization tests in mice. Low-dose EtOH $(0.5$ or $1 \mathrm{~g} / \mathrm{kg}$ twice/week)-induced CPP was stronger than that produced by saline control treatment, but the effect decreased with increasing numbers of conditioning trials. Moderate-dose/long-term EtOH exposure induced a weaker CPP than high-dose/short-term EtOH (2 g/ $/ \mathrm{kg}$ twice/week) exposure with the same total EtOH dose $(8 \mathrm{~g} / \mathrm{kg} / \mathrm{experiment})$. Acamprosate calcium, an anti-relapse drug, preclusively reduced EtOH-induced CPP. EtOH induced CPP and locomotor sensitization in black but not white chamber, although the initial preference and the basal locomotion in each chamber were equal. Therefore the brightness of the chamber had an effect on EtOHinduced sensitization. Moreover, additional studies indicated that EtOH-induced locomotor sensitization also depends on the dose but not the administration interval. Paired associative learning with EtOH exposure is a potent factor influencing the level of reward produced by EtOH. Moreover, exposure to high doses of alcohol, even on an intermittent schedule, carries a higher risk of addiction than exposure to moderate doses over longer periods.

Key words moderate alcohol (ethanol: EtOH); conditioned place preference (CPP); reward; sensitization; paired associative learning

Alcohol abuse is associated with serious physical and psychiatric illnesses, financial hardships, familial dysfunction, and violence, all of which place a substantial burden on society. Heavy alcohol consumption and long-term drinking increase the risk of alcohol-related diseases such as liver cirrhosis, cancer, pancreatitis, and depression. ${ }^{1,2)}$ In response, the World Health Organization (WHO) recently approved a Global Strategy to Reduce Harmful Use of Alcohol urging all countries to strengthen national responses to public health problems caused by alcohol use. ${ }^{3)}$ In Japan, 6.45 million people suffer from alcohol-related problems, with an estimated annual economic loss of 4.15 trillion yen associated with alcohol. ${ }^{4)}$ In December 2013, the Basic Act on Measures against Alcohol-related Health Harm was enacted in Japan, promoting education about drinking in moderation to prevent alcoholrelated problems.

Regular consumption of alcohol increases the propensity for addiction because of the rewarding effects of alcohol. Thus, many casual drinkers gradually increase total alcohol consumption, which becomes habitual over time. Although many studies in rodents have been conducted on chronic drinking and reward, ${ }^{5-7}$ previous studies have mainly focused on the rewarding effects of relatively high alcohol doses over short periods of time; therefore, the reward value of moderate and sustained drinking is not understood as well as that of shortterm heavy drinking. Thus, our primary research objective was to evaluate factors affecting the reward value of relatively low doses of alcohol and risk factors for increased drinking due to reward progression.

To identify factors affecting the reward value of moderate alcohol exposure and the concomitant risk of addiction, we employed conditioned place preference (CPP) testing and ethanol (EtOH)-induced locomotor sensitization in mice subjected to various moderate $\mathrm{EtOH}$ dosing regimens.

Following administration by intraperitoneal (i.p.) injection, alcohol is rapidly absorbed into the blood in a dose-dependent manner, after which it readily passes across the blood-brain barrier and reaches the brain. We therefore chose the i.p. route to clarify the relationship between alcohol dose and central neurological influence.

CPP is one of the most commonly used methods of testing the rewarding effects of drugs. Previous CPP studies have reported that EtOH $(2 \mathrm{~g} / \mathrm{kg}$ for 2 weeks, total dose of $8 \mathrm{~g} / \mathrm{kg})$ induced a preference for the chamber associated with its rewarding effects. ${ }^{8-11)}$ To assess the rewarding effects of lower doses of $\mathrm{EtOH}$, we administered $\mathrm{EtOH}(1 \mathrm{~g} / \mathrm{kg} /$ week) for 4 weeks (medium dose) or $\mathrm{EtOH}(0.5 \mathrm{~g} / \mathrm{kg} /$ week) for 8 weeks (low dose) for a total dose of $8 \mathrm{~g} / \mathrm{kg}$ in both groups. Furthermore, we used Acam, which suppresses alcohol reward preclusively following i.p. administration ${ }^{12)}$ and therapeutically following per os (p.o.) administration, ${ }^{13)}$ to confirm the reward values of the ethanol doses tested in our CPP experiments and examined the preclusive effects of Acamprosate calcium (Acam) on EtOH-induced reward.

Many abused drugs stimulate locomotor activity and produce sensitization, which is defined as an increase in the rein- 
forcement strength of a drug following repeated exposure. ${ }^{14-16)}$ Locomotor sensitization induced by activation of brain reward systems by alcohol is associated with alcohol-seeking behavior $^{17)}$ and an increased risk for developing alcoholism. Didone et al. have reported a significant positive correlation between sensitization of dopaminergic neuronal activity and behavioral sensitization induced by $\mathrm{EtOH}^{18)}$ Because the process of sensitization to the effects of a drug is affected by the administered dose, dosing interval, and administration term, ${ }^{19-23)}$ we also examined EtOH-induced locomotor sensitization under different levels of illumination in the CPP box, the dependence of EtOH-induced associative learning on the administered dose, and differences in the sensitizing effects of continuous and intermittent EtOH administration.

\section{MATERIALS AND METHODS}

Subjects Six-week-old DBA/2CrSlc male mice (Japan SLC, Inc., Hamamatsu, Japan) were acclimated to the animal facility for 2 weeks before testing. The animals were grouphoused (5 or 6 per cage) with ad libitum access to water and standard chow (CRF-1, Charles River Laboratories, Yokohama, Japan). The animal facility was maintained at $23 \pm 1^{\circ} \mathrm{C}$ with $55 \%$ humidity and a 12-h/12-h light/dark cycle (lights on from 7:30 a.m. to 7:30 p.m.).

We conducted all experiments from 9:00 to 16:30. All mice were enrolled in the experiment around the same time to allow adaptation to the circadian rhythms.

All experiments were performed in a room near by a breeding chamber. The brightness in the breeding cages varied with the height and position of the rack on which they were placed (front side brightness: 40 lux to 190 lux; back side brightness: less than 20 lux). More than $1 \mathrm{~h}$ before the beginning of the experiments in the morning or afternoon, the cages were moved from the breeding rack to a different rack (with a brightness less than approximately 140 lux) near the CPP boxes to calm the mice. After the experiments ended, the cages were moved back to the breeding racks.

All experiments were approved by the Institutional Animal Care and Use Committee of SAPPORO BREWERIES LTD. (permit numbers 2012-005, 2013-003, and 2014-003).

Apparatus We used a well-established CPP chamber with white and black compartments to evaluate learning associated with the rewarding properties of EtOH. ${ }^{24-27)}$ The CPP box ( $300 \mathrm{~mm} \times 150 \mathrm{~mm} \times 150 \mathrm{~mm}$; Brain Science Idea Inc., Osaka, Japan) consisted of 2 chambers, a black chamber and a white chamber, divided by a sliding door. The chambers were also distinguished by tactile cues; the black chamber had a smooth floor, whereas the white chamber had a textured floor. The CPP boxes were placed inside light- and sound-attenuating chambers equipped with a fan. Video camera was placed on the ceiling of each chamber to record behavior. Video was recorded and locomotor activity (staying time and locomotor distance in each area of the CPP chamber) was quantified using the ANY-maze Video Tracking System (Stoelting Co., Wood Dale, IL, U.S.A.). The illuminant conditions were 125 lux in the white chamber and off-scale low in the black chamber, which was covered with a black top.

Reagents EtOH was obtained from Wako Pure Chemical Industries, Ltd. (Osaka, Japan) as a special grade chemical. Acam for mouse injection was prepared from Regtect ${ }^{\circledR}$ (Nip- pon Shinyaku Co., Ltd., Kyoto, Japan) by micronization with a force mill, followed by sifting 3 times and suspension in water.

Experimental Design The protocols for Experiments 1, 3, and 5 are shown in Figs. 1A, 3A, and 5A, respectively. All CPP tests consisted of the following 4 phases: habituation, pre-test, conditioning, and post-test. Experiments 1 and 2 examined EtOH-dependent CPP without (Experiment 1) or with (Experiment 2) Acam pretreatment. Experiments 3, 4, and 5 examined locomotor sensitization in the same CPP chambers used for Experiments 1 and 2. In Experiment 3, EtOH-dependent CPP was simultaneously evaluated.

Habituation and Pre-Test In the habituation phase, each mouse was free to explore both CPP chambers (black and white) for $900 \mathrm{~s}$, once per day, for 3 consecutive days.

In the pre-test phase, each mouse was placed in the CPP box and allowed to explore both CPP chambers for $900 \mathrm{~s}$ while its movements were monitored. Movements were scored by both video tracking and a stopwatch to determine each mouse's initial (inherent) chamber preference ratio (black $v s$. white). Mice displaying an initial preference of $>800 \mathrm{~s}$ for 1 side were excluded from the experiment. Mice were then assigned to 8 groups in Experiment 1, 6 groups in Experiment 2, and 2 groups in Experiment 3 without significant differences in initial preference between the groups.

Conditioning Phases of Experiments 1 and 2 In the conditioning phase of Experiments 1 and 2, promptly after EtOH or saline administration, mice were allowed to enter only the white or black chamber for $300 \mathrm{~s}^{8}{ }^{828)}$ In both experiments, half of the mice in each group were conditioned in the white and black chambers in the order shown in Fig. 1A, and the other half of the mice were conditioned in the reverse order. In Experiment 1, we investigated how changes in the dose/term combination with equal overall alcohol dosage (dose pattern) affected CPP. Mice were assigned to 3 groups differing by dose/term (moderate-dose/long-term, medium-dose/mediumterm, or high-dose/short-term). The mice in each group underwent a counterbalanced conditioning procedure. Specifically, 106 mice were assigned to 8 groups, 4 white chamber groups and 4 black chamber groups, each receiving a different EtOH dose: $0 \mathrm{mg} / \mathrm{kg}$ (saline, $n=15), 0.5 \mathrm{~g} / \mathrm{kg} \quad(n=12), 1 \mathrm{~g} / \mathrm{kg} \quad(n=12)$, or $2 \mathrm{~g} / \mathrm{kg}(n=14)$. The administration terms were adjusted such that each group received the same total dose $(8 \mathrm{~g} / \mathrm{kg})$.

The EtOH $(2 \mathrm{~g} / \mathrm{kg})$ groups received 4 paired conditioning trials for 2 weeks (high-dose/short-term), the EtOH $(1 \mathrm{~g} /$ $\mathrm{kg}$ ) group received 8 paired conditioning trials for 4 weeks (medium-dose/medium-term groups), and the EtOH $(0.5 \mathrm{~g} / \mathrm{kg})$ group and saline group received 16 paired conditioning trials for 8 weeks (moderate-dose/long-term). A paired conditioning trial was conducted twice per week.

In Experiment 2, we examined the effect of Acam on EtOH-induced CPP in the black chamber. Mice $(n=84)$ were assigned to 6 groups (3 saline groups and $3 \mathrm{EtOH}$ groups).

Acam or water (vehicle control) was administered (p.o.) $1 \mathrm{~h}$ before saline or $\mathrm{EtOH}$ administration (i.p.). The 3 saline groups consisted of a group receiving water $(n=15)$, a group receiving Acam $(300 \mathrm{mg} / \mathrm{kg})($ Acam-300, $n=13)$, and a group receiving Acam $(600 \mathrm{mg} / \mathrm{kg})($ Acam-600, $n=12)$. Similarly, the $3 \mathrm{EtOH}$ groups consisted of a group receiving water $(n=17)$, a group receiving Acam-300 $(n=15)$, and a group receiving Acam-600 $(n=12)$. Promptly after saline or EtOH $(2 \mathrm{~g} / \mathrm{kg})$ 
administration, paired conditioning occurred (black or white chamber exploration, with EtOH conditioning only in the black chamber) for $300 \mathrm{~s}$ twice per week for 2 weeks.

Post-Test In the post-test phase of Experiments 1 and 2, each mouse was placed in the CPP box and allowed to explore the white and black chambers for $900 \mathrm{~s}$. Post-chamber preference was scored using video tracking and a stopwatch. The CPP value was calculated as the post-test value minus the pretest value for each mouse.

Locomotor Sensitization Tests Before the sensitization tests, each mouse was placed in the CPP box and allowed to explore the black and white chambers (Experiment 3) or only the black chamber (Experiments 4 and 5) for $900 \mathrm{~s}$ once per day for 3 consecutive days as habituation.

Locomotor activity was recorded separately (300s after EtOH or saline administration) in the black or white chamber. Because the top of the black chamber disrupts tracking, the top was removed and the illumination was set at 20 lux during tracking. A white patch was attached to the back of each mouse to enable tracking in the black chamber. To account for any potential stress associated with the patch, a dark-colored patch was attached to the back when the mouse was in the white chamber.

In Experiment 3, we investigated whether development of sensitization differs depending on the context of the reward. In the preliminary test, locomotor activity was measured for $300 \mathrm{~s}$ in the white chamber with a textured floor or a smooth floor (originally the black chamber floor). The next day, the floor of the white chamber was changed from textured to smooth to measure any inherent preference for either type of floor. In the primary test, locomotor activity (primary aim) and CPP (secondary aim) were measured in 24 mice simultaneously. The protocol for Experiment 3 is shown in Fig. 3A. On Days 1 and 2, each mouse was free to explore the black and white chambers of the CPP box for $900 \mathrm{~s}$ (habituation). Data from the second habituation day were used for the CPP pre-test. On days 3 and 6 , the chambers were closed using the removable guillotine door and locomotor activity was measured separately in both chambers for $900 \mathrm{~s}$ each. Twenty-four mice were assigned to the white-chamber group $(n=12)$ or black-chamber group $(n=12)$.

The white-chamber group received $\mathrm{EtOH}(2 \mathrm{~g} / \mathrm{kg})$ immediately before exploration of the white chamber and saline immediately before exploration of the black chamber, whereas the treatment before exploration of each chamber was reversed for the black-chamber group.

After administration of EtOH or saline, locomotor activity was recorded in the black or white chamber for $300 \mathrm{~s}$. Both groups received paired administration (saline before access to one chamber and EtOH before access to the other) twice per week for 4 weeks. Additionally, CPP post-tests were performed after the 2- and 4-weeks conditioning trials. The CPP value for each mouse was calculated as the post-test value minus the pre-test value.

In Experiment 4, the dose dependence of EtOH-induced sensitization was evaluated. Mice $(n=36)$ were assigned to the following 4 groups: saline $(n=9)$, $\mathrm{EtOH}(0.5 \mathrm{~g} / \mathrm{kg}, n=9), \mathrm{EtOH}$ $(1 \mathrm{~g} / \mathrm{kg}, n=9)$, and EtOH $(2 \mathrm{~g} / \mathrm{kg}, n=9)$. Each group received saline (i.p.) or EtOH (i.p.) every day for 16 d. After administration of EtOH or saline, locomotor activity was recorded in the black chamber for $300 \mathrm{~s}$.
In Experiment 5, we investigated whether the administration interval affected the development of sensitization by assessing the effects of consecutive and intermittent EtOH administration at the same daily dose. Mice $(n=36)$ were assigned to the following 4 groups: saline $(n=9), \mathrm{EtOH}(2 \mathrm{~g} /$ $\mathrm{kg}$ ) every day (daily EtOH group, $n=9)$, every $3 \mathrm{~d}(3-\mathrm{d} \mathrm{EtOH}$ group, $n=9)$, or every $6 \mathrm{~d}$ (6-d EtOH group, $n=9)$.

The protocol for Experiment 5 is shown in Fig. 5A. We performed the sensitization test 8 times by recording locomotion. The saline group and daily $\mathrm{EtOH}$ group received saline (i.p.) or $\mathrm{EtOH}(2 \mathrm{~g} / \mathrm{kg}$; i.p.) for $24 \mathrm{~d}$ (Days 1-24), the 3-d EtOH group received $\mathrm{EtOH}$ every $3 \mathrm{~d}$ for $24 \mathrm{~d}$ (first administration on Day 3 and eighth administration on Day 24), and the 6-d EtOH group received EtOH every $6 \mathrm{~d}$ for $48 \mathrm{~d}$ (first administration on Day 6 and eighth administration on Day 48).

To investigate the persistence of sensitization after EtOH withdrawal, the saline, daily $\mathrm{EtOH}$, and 3-d EtOH groups received $\mathrm{EtOH}(2 \mathrm{~g} / \mathrm{kg}$; i.p.) every $6 \mathrm{~d}$ after the first $24-\mathrm{d}$ regimen to monitor the washout effect for $48 \mathrm{~d}$. On Days 81 and 186, all groups received EtOH ( $2 \mathrm{~g} / \mathrm{kg}$; i.p.) or saline (salineonly group) and locomotor activity was recorded. Before withdrawal from $\mathrm{EtOH}$, mice in the daily $\mathrm{EtOH}$ group were injected with EtOH $(2 \mathrm{~g} / \mathrm{kg}) 30$ times, mice in the 3-d group were injected with EtOH 14 times, and mice in the 6-d group were injected with EtOH 10 times.

Data Analysis SPSS software 10.0.7 J for Windows (IBM Co., Armonk, NY, U.S.A.) was used for all statistical analyses. Data in the text and figures are presented as the mean \pm standard error of the mean (S.E.M.). Between-group comparisons between 2 groups (CPP values; black vs. white or saline vs. EtOH) were performed by unpaired $t$-tests (Figs. 1D, $\mathrm{E}, 3 \mathrm{C}$ ) and those between 3 or more groups were performed using two-way ANOVA (Figs. 2, 3C), or one-way followed by Tukey's post-hoc test (Figs. 1D, 2, 4A, 5C). Three-way ANOVA was applied for Figs. $1 \mathrm{~B}$ and $\mathrm{C}$ [treatment (saline or EtOH), and chamber (white and black), and time on CPP], Figs. 3D, E (group, epoch, and conditioning times on EtOHinduced locomotor activity), followed by the Bonferroni's post-hoc test. For locomotor sensitization, within-group comparisons were performed by one-way ANOVA followed by Dunnett's post-hoc test (vs. administration 1, Figs. 3B, 5B) or two-way ANOVA followed by the Bonferroni's post-hoc test (vs. administration 1) for multiple comparisons (Figs. 4B, 5C).

In all analyses, a $p$-value $<0.05$ was considered statistically significant.

\section{RESULTS}

Dose and Time Dependence of EtOH Administration on CPP To examine how the reward value of EtOH changes with dose and dosing term, we investigated the efficacy of specific dose/term combinations (e.g., high-dose/short-term vs. low-dose/long-term) with a constant total dose $(8 \mathrm{~g} / \mathrm{kg})$ in CPP induction.

In the pre-test phase, the average time spent in the white chamber $(n=106)$ was $437 \pm 11 \mathrm{~s}$ of the total $900 \mathrm{~s}$ exploration period (approximately 50\%), indicating no inherent preference for the white or black chamber. The administration regimens tested were EtOH $(2 \mathrm{~g} / \mathrm{kg}$ ) twice weekly for 2 weeks (highdose/short-term group), EtOH ( $1 \mathrm{~g} / \mathrm{kg})$ twice weekly for 4 weeks (medium-dose/medium-term group), and EtOH $(0.5 \mathrm{~g} /$ 
$\mathrm{kg}$ ) twice weekly for 8 weeks (moderate-dose/long-term group).

We examined the time-dependence of EtOH-induced CPP (Figs. 1B, C). In the EtOH $(1 \mathrm{~g} / \mathrm{kg})$ group, a threeway ANOVA was performed to assess the effects of treatment (saline or EtOH), chamber (white and black), and time
(2, 4 weeks) on CPP (Fig. 1B). This analysis resulted in a significant main effect of the chamber on the CPP score $[F(1,100)=6.95 ; p=0.010]$, but no significant effects of time $[F(1,100)=1.54 ;(p=0.217)]$ or treatment $[F(1,100)=0.238$; $(p=0.627)]$ were observed. There was a significant interaction between chamber and treatment $[F(1,100)=6.13 ;(p=0.015)]$,

\begin{tabular}{|c|c|c|c|c|c|c|c|c|c|c|c|c|c|c|}
\hline 8 groups & $\overline{D 1}$ & $\overline{D 2}$ & $\mathrm{D} 3$ & $\overline{D 4}$ & $\overline{D 5}$ & $\overline{D 6}$ & $\overline{D 7}$ & $\overline{D 8}$ & 89 & 010 & $\mathrm{D} 11$ & D12 & 013 & \\
\hline saline - black & $\overline{D n}$ & 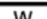 & 8 & W & & & & & & & & & & \\
\hline & Pre & W & $B$ & VV & $B$ & & & WV & $B$ & W & B & Post & & \\
\hline $0.5 \mathrm{~g} / \mathrm{kg}$ - black & Pre & $W$ & $B$ & $\mathrm{~W}$ & $B$ & & & $\mathrm{~W}$ & B & $\mathrm{W}$ & B & Post & & \\
\hline $1 \mathrm{~g} / \mathrm{kg}$ - black & Pre & W & B & W & $B$ & & & W & B & W & B & Post & & \\
\hline $2 \mathrm{~g} / \mathrm{kg}$ - black & Pre & W & B & W & B & & & $\mathrm{W}$ & B & $\mathrm{W}$ & B & Post & & \\
\hline saline - white & Pre & W & B & W & B & & & W & B & W & B & Post & & \\
\hline $0.5 \mathrm{~g} / \mathrm{kg}$ - white & Pre & W & B & W & $B$ & & & W & B & W & B & Post & & \\
\hline $1 \mathrm{~g} / \mathrm{kg}$ - white & Pre & $\bar{W}$ & $\bar{B}$ & $\bar{W}$ & $\bar{B}$ & & & $\mathrm{~W}$ & $\bar{B}$ & $\bar{W}$ & $\bar{B}$ & Post & & \\
\hline $2 \mathrm{~g} / \mathrm{kg}$ - white & Pre & $\mathrm{W}$ & B & $\bar{W}$ & B & & & $\mathrm{W}$ & B & $\mathrm{W}$ & $\bar{B}$ & Post & & \\
\hline & D15 & $\overline{D 16}$ & $\overline{\mathrm{D} 17}$ & D18 & D19 & $\mathrm{D} 20$ & $\overline{D 21}$ & $\mathrm{D} 22$ & $\mathrm{D} 23$ & D24 & $\overline{D 25}$ & D26 & D27 & D28 \\
\hline saline - black & W & $\mathrm{B}$ & W & B & & & & W & B & W & B & Post & & \\
\hline $0.5 \mathrm{~g} / \mathrm{kg}$ - black & $\bar{W}$ & $\bar{B}$ & $\bar{W}$ & $\bar{B}$ & & & & $\bar{W}$ & $\bar{B}$ & $\bar{W}$ & $\bar{B}$ & Post & & \\
\hline $1 \mathrm{~g} / \mathrm{kg}$ - black & W & B & W & B & & & & W & B & W & B & Post & & \\
\hline saline - white & $\bar{W}$ & 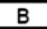 & $\bar{W}$ & B & & & & $\mathrm{W}$ & B & $\bar{W}$ & $\bar{B}$ & Post & & \\
\hline $0.5 \mathrm{~g} / \mathrm{kg}$ - white & $\bar{W}$ & B & $\bar{W}$ & B & & & & $\bar{W}$ & B & $\bar{W}$ & B & Post & & \\
\hline $1 \mathrm{~g} / \mathrm{kg}$ - white & W & B & W & B & & & & W & B & W & B & Post & & \\
\hline & D29 & D30 & D31 & D32 & D33 & D34 & D35 & D36 & D37 & D38 & D39 & D40 & D41 & D42 \\
\hline saline - black & $W$ & $\mathrm{~B}$ & $\mathrm{~W}$ & $\mathrm{~B}$ & & & & $\mathrm{~W}$ & $\mathrm{~B}$ & $w$ & $\mathrm{~B}$ & & & \\
\hline $0.5 \mathrm{~g} / \mathrm{kg}$ - black & W & $\bar{B}$ & $\bar{W}$ & $\bar{B}$ & & & & $\bar{W}$ & $\bar{B}$ & $\bar{W}$ & $\bar{B}$ & & & \\
\hline saline - white & W & $B$ & W & $B$ & & & & W & B & W & B & & & \\
\hline $0.5 \mathrm{~g} / \mathrm{kg}$ - white & $\mathrm{W}$ & $\bar{B}$ & $\bar{W}$ & $\bar{B}$ & & & & $\bar{W}$ & $\bar{B}$ & $\mathrm{~W}$ & $\bar{B}$ & & & \\
\hline & D43 & D44 & D45 & D46 & D47 & D48 & $\mathrm{D} 49$ & D50 & D51 & $\mathrm{D} 52$ & D53 & D54 & & \\
\hline saline - black & $W$ & $\mathrm{~B}$ & W & $\mathrm{B}$ & & & & $\mathrm{W}$ & $\mathrm{B}$ & W & $\mathrm{B}$ & Post & & \\
\hline $0.5 \mathrm{~g} / \mathrm{kg}$ - black & W & B & W & $B$ & & & & W & B & W & $B$ & Post & & \\
\hline saline - white & W & B & W & B & & & & W & B & W & B & Post & & \\
\hline $0.5 \mathrm{~g} / \mathrm{kg}$ - white & $\mathrm{W}$ & $\bar{B}$ & W & $\bar{B}$ & & & & W & B & W & $\mathrm{B}$ & Post & & \\
\hline
\end{tabular}
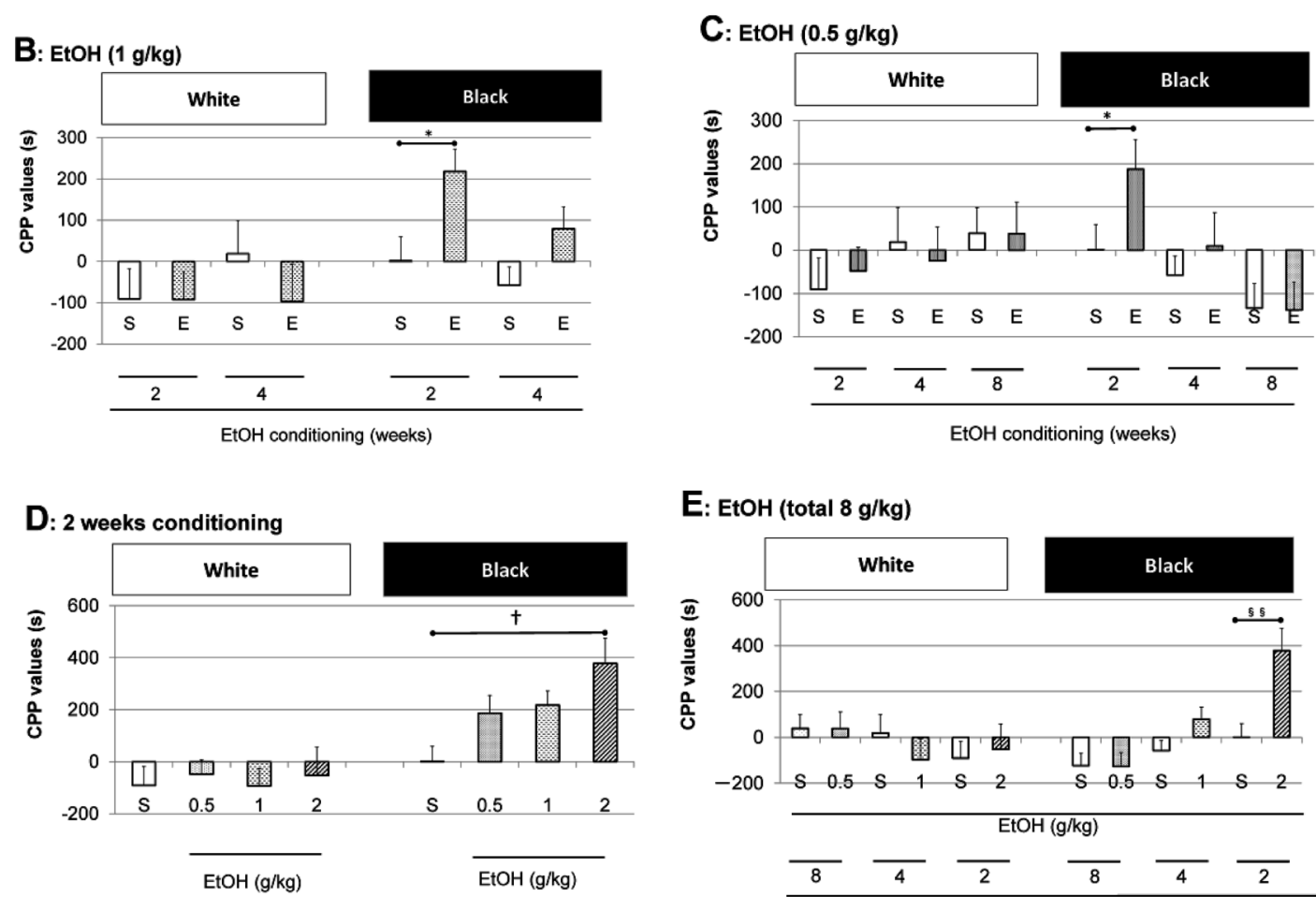

\section{E: EtOH (total $8 \mathrm{~g} / \mathrm{kg}$ )}

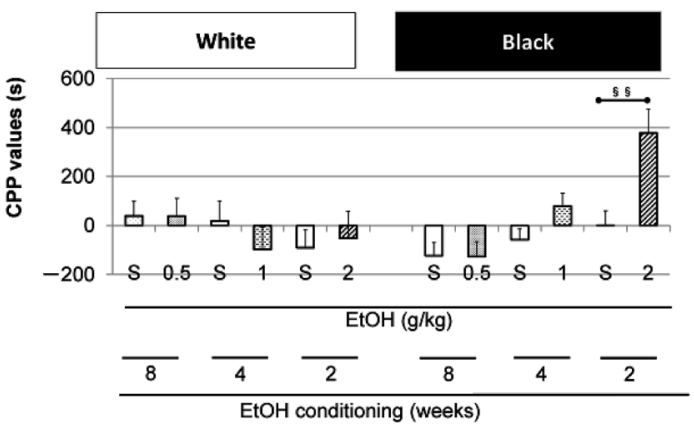

Fig. 1. Dose- and Time-Dependence of EtOH-Induced CPP in the White and Black Chambers (Experiment 1)

The EtOH $(0.5 \mathrm{~g} / \mathrm{kg})$ groups (white: $n=12$; black: $n=12)$ received 16 conditioning trials over 8 weeks, the EtOH $(1 \mathrm{~g} / \mathrm{kg})$ groups $($ white: $n=12 ;$ black: $n=12)$ received 8 conditioning trials over 4 weeks, and the EtOH ( $2 \mathrm{~g} / \mathrm{kg}$ ) groups (white: $n=14$; black: $n=14$ ) received 4 conditioning trials over 2 weeks. The saline groups (white: $n=15$; black: $n=15$ ) received conditioning trials 2 times per week. Each group was subjected to post-test once per 2 weeks maximum for up to 8 weeks. (A) Protocol for Experiment 1 . Half of the mice in each group were conditioned in the white and black chambers in that order, whereas the other half of the mice were conditioned in the reverse order. (B, C) Time-dependence of EtOH $(1 \mathrm{~g} / \mathrm{kg}, \mathrm{B})$ or $(0.5 \mathrm{~g} / \mathrm{kg}, \mathrm{C})$-induced CPP at indicated weeks. (D) Dose-dependence of EtOH-induced CPP at 2 weeks. (E) Comparison of dose/term combinations in EtOH $(8 \mathrm{~g} / \mathrm{kg})$-induced CPP. ${ }^{*} p<0.05$, three-way ANOVA followed by the Bonferroni's post-hoc test. ${ }^{\dagger} p<0.05$, one-way ANOVA followed by Tukey's post-hoc test. ${ }^{\S} p<0.01$, unpaired $t$-test. D, day; Pre, pre-test; Post, post-test. W, conditioning in the white chamber for $300 \mathrm{~s}$; B, conditioning in the black chamber for $300 \mathrm{~s}$; S, saline; E, ethanol. 
but no interaction between chamber and time $[F(1,100)=2.53$; $p=0.115]$, treatment and time $[F(1,100)=1.03 ; p=0.312]$, or all 3 factors $[F(1,100)=0.032 ; p=0.859]$. Using the Bonferroni's post-hoc test, a significant difference was identified between the CPP scores of the saline and EtOH groups in the black chamber. There was a significant difference between the CPP scores of the saline and EtOH groups in the black chamber at 2 weeks $(p=0.025)$, but not at 4 weeks $(p=0.153)$.

In the EtOH $(0.5 \mathrm{~g} / \mathrm{kg})$ group, a three-way ANOVA was performed to assess the effects of treatment (saline or EtOH), chamber (white and black), and time (2, 4, and 8 weeks) on CPP (Fig. 1C). There were no significant main effects of chamber $[F(1,148)=0.078, p=0.780]$, treatment $[F(1,148)=1.16, p=0.284]$, or time $[F(2,148)=0.841, p=0.433]$ on CPP score. There was a significant interaction of chamber and time $[F(2,148)=6.41, p=0.002]$, but no significant interaction of chamber and treatment $[F(1,148)=1.17, p=0.281]$, treatment and time $[F(2,148)=0.917 ; p=0.402]$, or all 3 factors $[F(2,148)=0.331 ; p=0.718]$. Using the Bonferroni's post-hoc test, a significant difference was identified between the CPP scores of the saline and EtOH groups in the black chamber at 2 weeks $(p=0.049)$, but not at 4 weeks $(p=0.472)$ or 8 weeks $(p=0.964)$.

The EtOH dose (1 or $0.5 \mathrm{~g} / \mathrm{kg}$ )-induced CPP was stronger than that produced by the saline treatment, but the effect decreased with increasing numbers of conditioning trials.

We next examined the dose-dependence of CPP over a constant duration of administration (Fig. 1D) using twoway ANOVA (EtOH dose and chamber). The chamber $[F(1$, $96)=24.7 ; p<0.001]$ and $\mathrm{EtOH}$ dose $[F(3,96)=2.72 ; p=0.049]$ had significant effects on CPP, but there was no interaction between the chamber and EtOH dose $[F(3,96)=1.88 ; p=0.138]$. We performed a one-way ANOVA followed by Tukey's post-hoc test to confirm the main effect (EtOH dose) in each chamber. In the black chamber, the CPP value showed a dosedependent increase in comparison with that of the matched saline group, but this difference was only significantly different for the EtOH $(2 \mathrm{~g} / \mathrm{kg})$ group $(p=0.002)$ compared to EtOH $(0.5 \mathrm{~g} / \mathrm{kg})(p=0.416)$ and EtOH $(1 \mathrm{~g} / \mathrm{kg})(p=0.264)$ groups. The CPP scores of the EtOH $(0.5 \mathrm{~g} / \mathrm{kg})(p=0.047)$ and EtOH $(1 \mathrm{~g} / \mathrm{kg})$ groups $(p=0.013)$ differed significantly from that of the matched saline group, as determined by unpaired $t$-test. The same conditioning paradigm in the white chamber did not yield any significant differences between the CPP scores of the EtOH and matched saline control groups after 2 weeks $(p>0.05)$. Thus, EtOH CPP was dose-dependent. Moreover, lower EtOH doses have a measurable reward value.

We summarized the results of dose/time (EtOH $2 \mathrm{~g} / \mathrm{kg} / 2$ weeks, EtOH $1 \mathrm{~g} / \mathrm{kg} / 4$ weeks, and $\mathrm{EtOH} 0.5 \mathrm{~g} / \mathrm{kg} / 8$ weeks) effect on CPP with the same total $\mathrm{EtOH}(8 \mathrm{~g} / \mathrm{kg})$ dose in the black and white chamber groups (Fig. 1E). Conditioning to the white chamber with a textured floor cue did not result in significant CPP induction in any of the EtOH groups in comparison with that of the matched saline group (high-dose/ short-term, $p=0.764$; medium group, $p=0.349$; moderate-dose/ long-term, $p=0.996$ ), indicating that the tested $\mathrm{EtOH}$ regimens had weak reward efficacy (Fig. 1E). Conditioning to the black chamber with a smooth floor cue resulted in significant CPP, but only in the high-dose/short-term group $(p=0.002)$ and not in the medium $(p=0.057)$ or moderate-dose/long-term group $(p=0.960)$ (Fig. 1E). These results suggest that higher doses for shorter periods are more rewarding than lower doses for longer periods, even when the total amount administered is equal. On post-test days, there was no significant difference in the average body weight of the $\mathrm{EtOH}$ and saline groups (saline group at 2 weeks: $24.2 \pm 0.3 \mathrm{~g} ; 2 \mathrm{~g} / \mathrm{kg} \mathrm{EtOH}$ group at 2 weeks: $23.6 \pm 0.3 \mathrm{~g}$; saline group at 4 weeks: $25.3 \pm 0.3 \mathrm{~g} ; 1 \mathrm{~g} / \mathrm{kg}$ EtOH group at 4 weeks: $25.2 \pm 0.3 \mathrm{~g}$; saline group at 8 weeks: $27.0 \pm 0.3 \mathrm{~g} ; 0.5 \mathrm{~g} / \mathrm{kg}$ EtOH group at 8 weeks: $27.5 \pm 0.3 \mathrm{~g}$ ).

Furthermore, we examined whether the CPP values were different between the EtOH-conditioning orders of whiteblack (shown in Fig. 1A) and black-white (the reverse order) in Fig. 1E. There were no significant differences in CPP values of both white EtOH-conditioning groups (EtOH 0.5, 1 , and $2 \mathrm{~g} / \mathrm{kg} ; p=0.854, p=0.846$, and $p=0.623$, respectively) and black EtOH-conditioning groups (EtOH 0.5, 1, and $2 \mathrm{~g} / \mathrm{kg}$; $p=0.525, p=0.383$, and $p=0.231$, respectively).

Effects of Acam on EtOH-Induced CPP To confirm the rewarding value of EtOH administered during our CPP protocol, we investigated whether Acam blocked EtOH-induced CPP (Fig. 2). There were no significant differences in the initial average time spent in the white chamber among the 6 experimental groups $(p=0.293)$. We performed a two-way ANOVA to assess the effects of pretreatment (water or Acam) and treatment (saline or EtOH) on CPP. There were significant main effects of pretreatment $[F(2,78)=8.94 ; p<0.001]$ and treatment $[F(1,78)=14.9 ; p<0.001]$, but no interaction between pretreatment and treatment $[F(2,78)=1.25 ; p=0.292]$. Tukey's post-hoc analysis indicated that the CPP values of the 3 saline groups (water, Acam-300, and Acam-600) differed significantly from that of the EtOH $(2 \mathrm{~g} / \mathrm{kg})$ group pre-treated with water $(p<0.05)$ and did not differ among the 3 saline groups $(p>0.05)$.

Furthermore, the CPP scores of the $\mathrm{EtOH}(2 \mathrm{~g} / \mathrm{kg})$ plus Acam-300 group and EtOH $(2 \mathrm{~g} / \mathrm{kg})$ plus Acam-600 group were not significantly different from those of the 3 saline

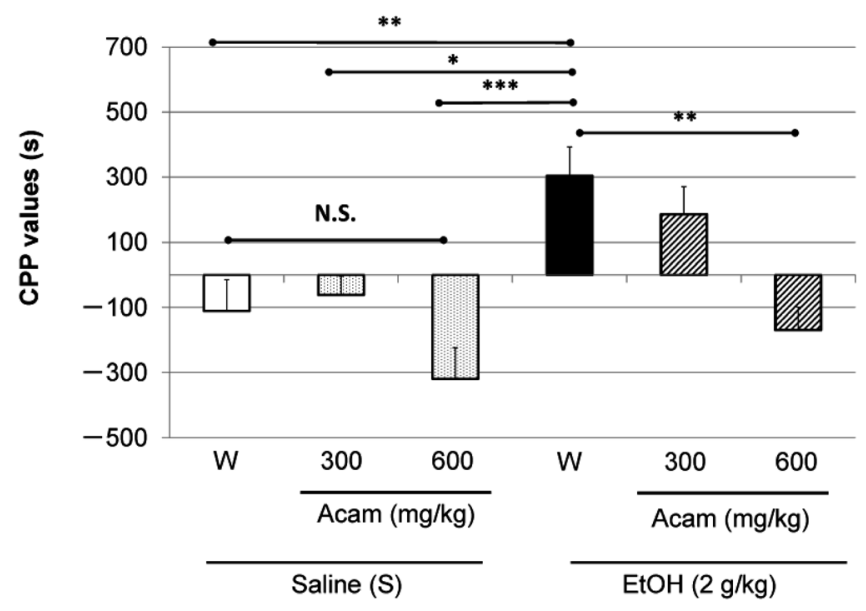

Fig. 2. Effect of Acamprosate Calcium (Acam) on EtOH-Induced CPP in the Black Chamber (Experiment 2)

Acam or water (W: vehicle control) was administered (p.o.) $1 \mathrm{~h}$ before saline or EtOH administration (i.p.). The 3 saline groups consisted of a water group $(n=15)$ and groups that received Acam $(300 \mathrm{mg} / \mathrm{kg}, n=13)$ and Acam $(600 \mathrm{mg} / \mathrm{kg}, n=12)$. Similarly, the $3 \mathrm{EtOH}$ groups consisted of a water group $(n=17)$, a group that received Acam $(300 \mathrm{mg} / \mathrm{kg}, n=15)$, and a group that received Acam $(600 \mathrm{mg} / \mathrm{kg}$, $n=12)$. After saline or EtOH $(2 \mathrm{~g} / \mathrm{kg})$ administration, conditioning was performed in 2 sets (saline twice + EtOH twice) per week for 2 weeks. Half of the mice in each group were conditioned in the white and black chambers in that order, whereas the other half of the mice were conditioned in the reverse order. ${ }^{*} p<0.05,{ }^{*} p<0.01$, $* * * p<0.001$, one-way ANOVA followed by Tukey's post-hoc test. N.S., not significant. 
groups $(p>0.05)$. Moreover, Acam-600 significantly blocked the development of CPP associated with EtOH $(2 \mathrm{~g} / \mathrm{kg})$ in comparison with vehicle $(p=0.002)$.

Development of Sensitization and Place Preference to EtOH in the Black and White Chambers In the next experiment, we examined whether EtOH-induced CPP and locomotor sensitization were altered by specific aspects of the conditioning environment (context). As shown previously (Fig. 1D), EtOH (2 $\mathrm{g} / \mathrm{kg})$ produced CPP most effectively when it was paired with the black chamber. In the preliminary test for Experiment 3, examination of the effects of floor characteristics in the white chamber revealed that total locomotor activity over a period of $300 \mathrm{~s}$ was $7.3 \pm 0.3 \mathrm{~m}$ with a textured floor and $8.0 \pm 0.6 \mathrm{~m}$ with a smooth floor $(p=0.339)$.

As with CPP induction, illumination also had a significant influence on sensitization. The primary test of Experiment 3 revealed that the progressive increase in locomotor activity induced by repeated EtOH exposure was affected by chamber brightness. Before EtOH administration, the basal locomo- tor activity of mice in the black chamber was significantly greater than that of mice in the white chamber $(14.7 \pm 0.8 \mathrm{~m}$ vs. $5.7 \pm 1.3 \mathrm{~m}, p<0.001$; Fig. $3 \mathrm{~B})$. After the first EtOH administration, there was no significant difference in the locomotor activity levels of the mice in the white and black chambers $(p=0.882)$. During the 8 EtOH conditioning trials (Con1Con8), two-way ANOVA revealed that EtOH-induced locomotion was significantly affected by chamber illumination $[F(1$, $173)=65.2 ; p<0.01]$ and EtOH administration duration $[F(7$, $173)=4.31 ; p<0.001]$, with no interaction between illumination and duration $[F(7,173)=1.10 ; p>0.05]$ (Fig. 3B).

Sensitization (defined as a significant increase in locomotion distance upon EtOH exposure after an initial EtOH trial (Con1)) was evaluated using one-way ANOVA followed by Dunnett's post-hoc test (vs. Con1). Locomotor activity in the dark chamber was significantly greater at Con5 $(p=0.049)$, Con7 $(p=0.001)$, and Con8 $(p<0.001)$ than locomotor activity at Con1. However, in the white chamber, there was no significant change in locomotion distance after Con1 $(p=0.138)$ (Fig.

\section{A: Experiment 3}

\begin{tabular}{|c|c|c|c|c|c|c|c|c|c|c|c|c|}
\hline & & & & & & & & & & & & \\
\hline & & & & & & & & & & & & \\
\hline \multirow{2}{*}{2 groups } & $\mathrm{H}$ & Pre & $\mathrm{BL}(\mathrm{W})$ & & & & & & & & & \\
\hline & D6 & D7 & D8 & D9 & D10 & D11 & D12 & D13 & D14 & D15 & D16 & D17 \\
\hline \multirow[t]{2}{*}{2 groups } & $B L(B)$ & $w$ & $B$ & $W$ & $B$ & & & $w$ & $B$ & $w$ & $\mathrm{~B}$ & Post \\
\hline & D18 & D19 & D20 & D21 & D22 & $\mathrm{D} 23$ & D24 & D25 & D26 & D27 & D28 & D29 \\
\hline 2 groups & W & $B$ & W & B & & & & W & $B$ & W & B & Post \\
\hline
\end{tabular}

\section{B: Development of Sensitization}

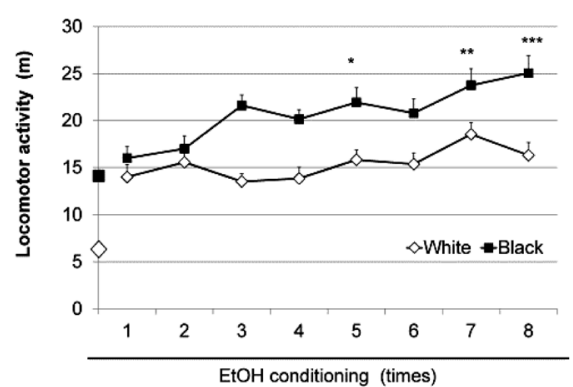

D: White Chamber

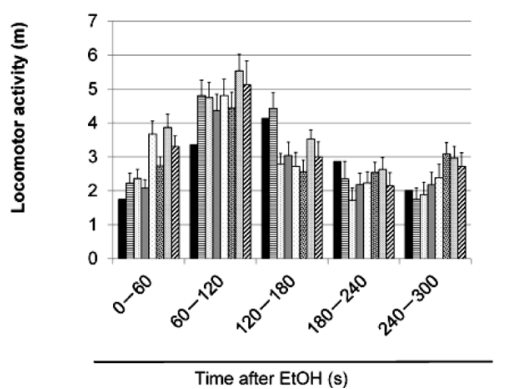

C: CPP

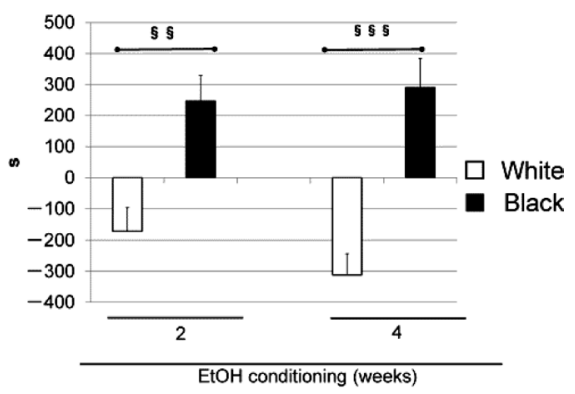

E: Black Chamber

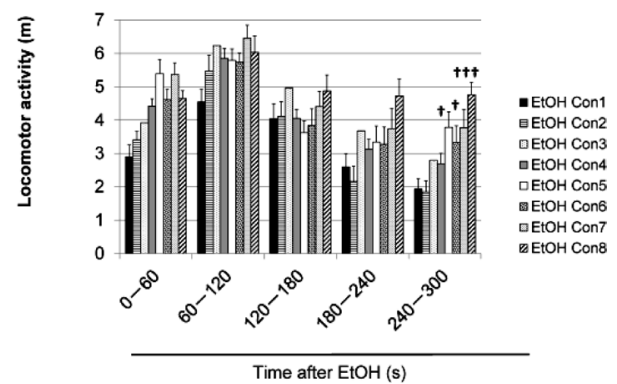

Fig. 3. Time-Dependence of Sensitization and Place Preference to $2 \mathrm{~g} / \mathrm{kg}$ EtOH in the Black and White Chambers (Experiment 3)

Mice in both groups (white: $n=12$; black: $n=12)$ alternatively received saline or EtOH $(2 \mathrm{~g} / \mathrm{kg}$ ) as in the CPP procedure. Mice received EtOH ( $2 \mathrm{~g} / \mathrm{kg}$; i.p.) 8 times during 4 weeks conditioning phase, and EtOH-induced locomotion was measured every $60 \mathrm{~s}$ for $300 \mathrm{~s}$ just after the injection. Post test of CPP were performed at 2 and 4 weeks after the beginning of the conditioning. (A) Protocol for Experiment 3. H, habituation (900 s): Pre, pre-test for CPP at the 2nd habituation; Post, post-test: BL(W), basal locomotion in the white chamber: BL(B), basal locomotion in the black chamber. W, conditioning in the white chamber for $300 \mathrm{~s}$; B, conditioning in the black chamber for 300 s. (B) Development of locomotor sensitization to EtOH. (C) Maintenance of CPP induced by EtOH ( $2 \mathrm{~g} / \mathrm{kg})$ in the black and white chambers. (D, E) Time-dependent changes in the EtOH-induced locomotion in the black (D) or white (E) chamber at each conditioning day. $* p<0.05, * * p<0.01$, $* * * p<0.001 v s$. EtOH conditioning 1 , oneway ANOVA followed by Dunnett's post-hoc test. ${ }^{\S \S} p<0.01,{ }^{\S \S} p<0.001$, unpaired $t$-test. ${ }^{\dagger} p<0.05$, ${ }^{\dagger \dagger} p<0.001 v s$. that at $240-300 \mathrm{~s}$ of EtOH conditioning 1 , three-way ANOVA followed by the Bonferroni's post-hoc test. D, day; Con, conditioning. 
3B) indicating a lack of sensitization in the white chamber and underscoring the dominant influence of illumination on EtOHinduced CPP.

In the CPP pre-test phase at the 2nd habituation, the average time spent by the EtOH $(2 \mathrm{~g} / \mathrm{kg})$ groups in the white and black chambers was $485 \pm 37 \mathrm{~s}(n=12)$ and $493 \pm 24 \mathrm{~s}(n=12)$, respectively (of the total 900 s exploration period). In the CPP values (Fig. 3C), a two-way ANOVA was performed to assess the effects of EtOH conditioning chamber color (black or white) and duration (2- or 4-weeks) on CPP values. There was a significant main effect of color $[F(1,44)=40.5 ; p<0.001]$, but no main effect of duration $[F(1,44)=0.302 ; p=0.550]$ or
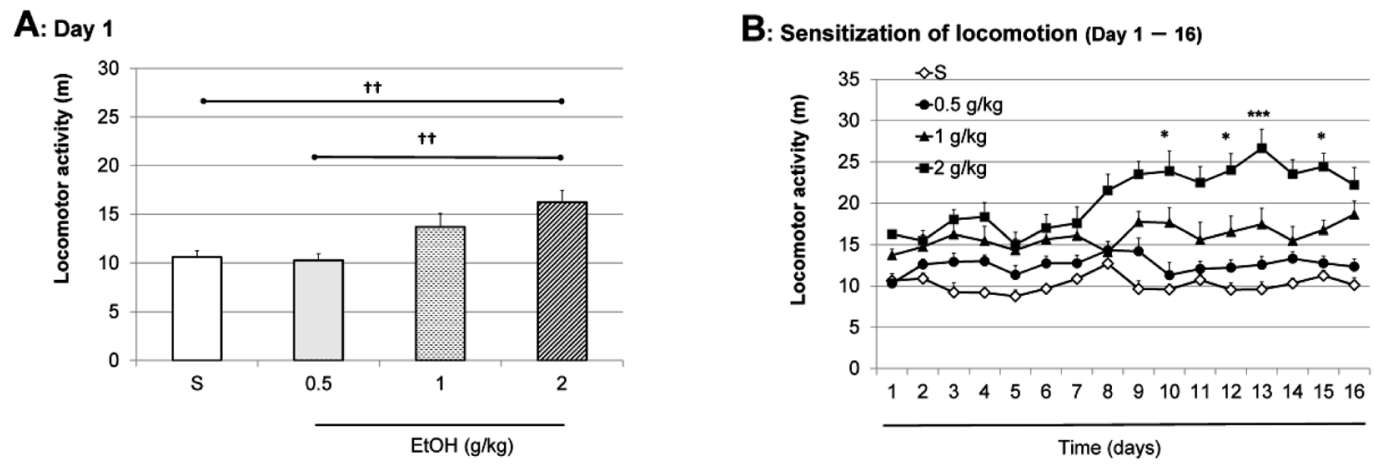

Fig. 4. Dose-Dependence of EtOH-Induced Locomotor Sensitization (Experiment 4)

Mice ( $n=36)$ were assigned to the following 4 groups: saline ( $n=9)$, EtOH ( $0.5 \mathrm{~g} / \mathrm{kg}, n=9), \mathrm{EtOH}(1 \mathrm{~g} / \mathrm{kg}, n=9)$, and EtOH ( $\mathrm{g} / \mathrm{kg}, n=9)$. Saline, EtOH 0.5 g/kg, $1 \mathrm{~g} / \mathrm{kg}, \mathrm{or}$ $2 \mathrm{~g} / \mathrm{kg}$ was administered (i.p.) for 16 consecutive days. (A) Dose-dependence of EtOH-induced locomotion after administration 1. (B) Dose dependence of EtOH-induced locomotor sensitization. ${ }^{\dagger} p<0.01$, one-way ANOVA followed by Tukey's post-hoc test. $* p<0.05, * * * p<0.001 v s$. EtOH Day 1 , two-way ANOVA followed by the Bonferroni's post-hoc test $v s$. administration 1.

\begin{tabular}{|c|c|c|c|c|c|c|c|c|c|c|c|c|c|c|c|c|c|c|}
\hline \multicolumn{19}{|c|}{ A: Experıment 5} \\
\hline & 01 & 02 & U. & & U5 & & & & & & & & & & & & & 018 \\
\hline saline & B & B & B & B & B & B & B & B & B & B & B & B & B & B & B & B & B & B \\
\hline EtOH every day & $B$ & $B$ & B & B & B & B & $B$ & $B$ & $B$ & $B$ & B & B & B & B & B & $B$ & $B$ & $B$ \\
\hline EtOH every 3 days & & & B & & & B & & & B & & & B & & & B & & & B \\
\hline EtOH every 6 days & & & & & & $\mathrm{B}$ & & & & & & $\mathrm{B}$ & & & & & & $B$ \\
\hline 4 groups & D19 & $\mathrm{D} 20$ & $\mathrm{D} 21$ & $\mathrm{D} 22$ & $\mathrm{D} 23$ & D24 & $\mathrm{D} 25$ & $\mathrm{D} 26$ & D27 & $\mathrm{D} 28$ & $\overline{\mathrm{D} 29}$ & $\mathrm{D} 30$ & D31 & D32 & D33 & D34 & D35 & D36 \\
\hline saline & $\mathrm{B}$ & $\mathrm{B}$ & $\mathrm{B}$ & $\mathrm{B}$ & $\mathrm{B}$ & $\mathrm{B}$ & & & & & & $\mathrm{B}$ & & & & & & $\mathrm{B}$ \\
\hline EtOH every day & $B$ & $B$ & B & B & B & $B$ & & & & & & $\bar{B}$ & & & & & & $\bar{B}$ \\
\hline EtOH every 3 days & & & B & & & B & & & & & & B & & & & & & B \\
\hline EtOH every 6 days & & & & & & $B$ & & & & & & $\mathrm{~B}$ & & & & & & $\mathrm{~B}$ \\
\hline & D37 & $\mathrm{D} 38$ & D39 & D40 & D41 & D42 & D43 & D44 & D45 & $\mathrm{D} 46$ & D47 & $\mathrm{D} 48$ & & D81 & & $\mathrm{D} 186$ & & \\
\hline saline & & & & & & $\mathrm{B}$ & & & & & & $\mathrm{B}$ & & $\mathrm{B}$ & & $\mathrm{B}$ & & \\
\hline EtOH every day & & & & & & B & & & & & & B & & B & & B & & \\
\hline $\mathrm{EtOH}$ every 3 days & & & & & & $B$ & & & & & & $B$ & & $\mathrm{~B}$ & & $\mathrm{~B}$ & & \\
\hline EtOH every 6 days & & & & & & $\mathrm{B}$ & & & & & & $\bar{B}$ & & $\mathrm{~B}$ & & $\mathrm{~B}$ & & \\
\hline
\end{tabular}
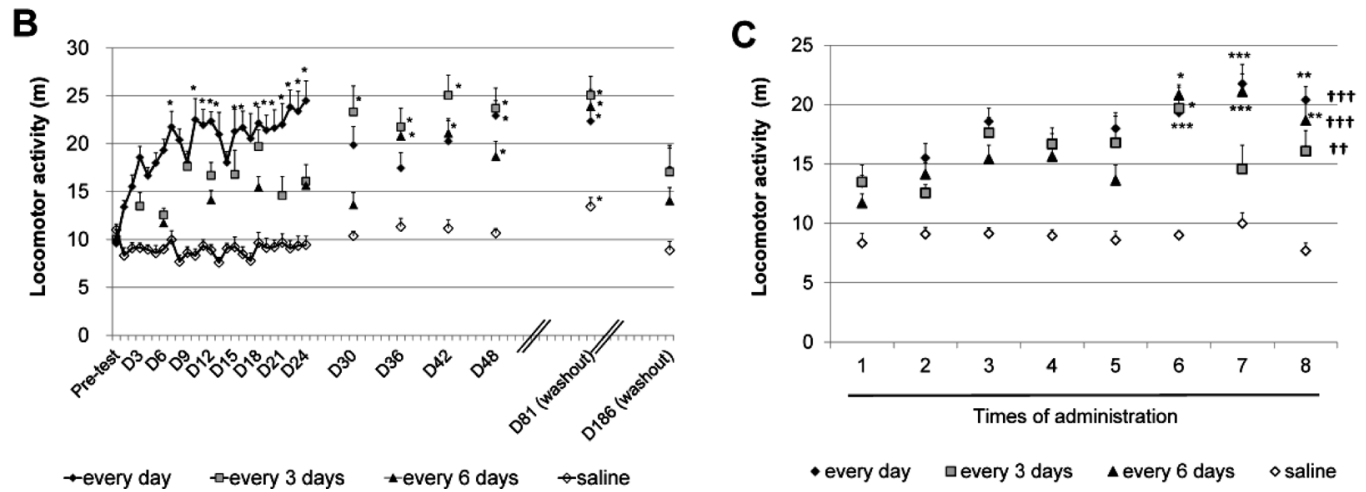

Fig. 5. Effect of Different Intervals of EtOH ( $2 \mathrm{~g} / \mathrm{kg})$ Administration on Locomotor Sensitization

Until Day 24, mice in the daily EtOH group $(n=9)$ received EtOH every day, those in the 3-d EtOH group $(n=9)$ every $3 \mathrm{~d}$, and those in EtOH 6-d group $(n=9)$ every $6 \mathrm{~d}$. Thereafter, all groups received EtOH every $6 \mathrm{~d}$ until Day 48, and EtOH administration to all mice was discontinued until Day 80 . Mice in the saline group received saline on the schedule to treat the daily group. Note some mice died after Day 48 (see text). Locomotor activity was recorded for $300 \mathrm{~s}$ just after the injection of EtOH (2g/kg; i.p.) or saline (saline-only group). (A) Protocol for Experiment 5. B: Locomotor measurement in the black chamber for 300 s. Gray color boxes: the eighth administration in each group. (B) Changes of EtOH-induced locomotor sensitization during 8 months after the first EtOH administration. * $p<0.05 v s$. EtOH administration 1 , one-way ANOVA followed by Dunnett's post-hoc test. (C) Comparison between daily and intermittent EtOH over the first 8 administrations. EtOH-induced locomotion every day from Days 1 to 8 in the saline and daily EtOH groups, from Days 3 to 24 in the 3-d EtOH group, and from Days 6 to 48 in the 6-d EtOH group are shown. Note EtOH induced sensitization in 8 rounds of exposure in all EtOH groups. ${ }^{*} p<0.05, * * p<0.01, * * * p<0.001 v s$. EtOH administration 1, two-way ANOVA followed by the Bonferroni's post hoc test $v s$. administration $1 .{ }^{\dagger} p<0.01,{ }^{\dagger \dagger} p<0.001$ vs. saline group, one-way ANOVA followed by Tukey's post-hoc test. D, day. 
interaction of color and duration $[F(1,44)=1.32 ; p=0.257]$. The EtOH-induced CPP value of the group conditioned in the black chamber was significantly higher than that of the group conditioned in the white chamber ( 2 weeks, $p=0.001 ; 4$ weeks, $p<0.001$; unpaired $t$-test). No saline group was included in this experiment.

Locomotor activity was not constant over the exploratory term (Figs. 3D, E) because peak locomotor activity was highest during the 60-120s period and decreased thereafter. Peak ambulation in the black chamber was higher than that in the white chamber within this early period. Moreover, the reduction in locomotion with time was smaller in later conditioning trials in the black chamber. A three-way ANOVA to assess the influence of group, epoch, and conditioning time on EtOH-induced locomotor activity demonstrated significant main effects of group $[F(1,866)=136.6 ; p<0.001]$, epoch $[F(4$, $866)=91.1 ; p<0.001]$, and conditioning time $[F(7,866)=10.4$; $p<0.001$ ], as well as significant interactions between epoch and conditioning time $[F(28,866)=2.71 ; p<0.001]$ and group and conditioning time $[F(7,866)=3.83 ; p<0.001]$. There was no interaction for group and epoch $[F(4,866)=2.30 ; p=0.058]$ or group, epoch, and conditioning time $[F(28,866)=0.787$; $p=0.778]$.

Using the Bonferroni's post-hoc test to compare locomotor activity among the mice at each conditioning trial during the $240-300 \mathrm{~s}$ epoch revealed that EtOH-induced locomotor activity at Con1 differed significantly from activity at Con5 $(p=0.043)$, Con7 $(p=0.045)$, and Con8; $p<0.001)$ (Fig. 3E), but no difference in the locomotor activity measured at Con1 in the white chamber and that measured at the other trials was observed $(p>0.05)$ (Fig. 3D). The greater locomotor activity during the 240-300s epoch at Con5, Con7, and Con8 may have contributed to the longer total locomotor distance measured over the full $300 \mathrm{~s}$ epoch.

These results suggest that EtOH-induced locomotor sensitization and CPP are affected predominantly by a illumination, and not by the tactile sensation associated with the test chamber (floor texture).

Dose Dependence of EtOH-Induced Locomotor Sensitization We also examined the dose-dependence of EtOH locomotor sensitization under daily EtOH administration. Consistent with the results shown in Fig. 1D, there was a dose-dependent increase in locomotion after the first EtOH administration (Fig. 4A), upon which locomotor activity in the EtOH $(2 \mathrm{~g} / \mathrm{kg})$ group was significantly higher than that of the saline group $(p=0.007)$ and $\mathrm{EtOH}(0.5 \mathrm{~g} / \mathrm{kg})$ group $(p=0.003)$ (one-way ANOVA followed by Tukey's post-hoc test). We examined the main effects of EtOH doses and duration using two-way ANOVA. EtOH-induced locomotion was significantly affected by EtOH dose $[F(3,459)=140.61 ; p<0.001]$ and EtOH administration duration $[F(15,459)=2.97 ; p<0.001]$, with an interaction between dose and duration $[F(45,523)=1.56$; $p=0.014]$. In general, daily administration of lower EtOH doses induced little sensitization. Significant sensitization was only observed in the EtOH $(2 \mathrm{~g} / \mathrm{kg})$ group on Days $10,12,13$, and 15 ( $p=0.046,0.025,<0.001$, and 0.011 , respectively; Bonferroni's post-hoc test vs. Day 1) (Fig. 4B).

Effect of EtOH Administration Interval on Sensitization Next, we examined the impact of changes in the conditioning interval on the development of sensitization using a single dose of EtOH (Fig. 5).
We examined both the effect of different intervals of EtOH ( $2 \mathrm{~g} / \mathrm{kg}$ ) administration (saline, daily EtOH, EtOH every $3 \mathrm{~d}$, and $\mathrm{EtOH}$ every $6 \mathrm{~d}$ ) on locomotor sensitization and the rate at which sensitization disappeared after withdrawal from EtOH using one-way ANOVA followed by Dunnett's post-hoc test (vs. Administration 1) (Fig. 5B).

In the daily EtOH group, locomotor activity was significantly different $v s$. administration 1 (Day 1) on Day 7 $(p=0.019)$, Day $10(p=0.007)$, Day $11(p=0.022)$, Day 12 $(p=0.009)$, Day $13(p=0.049)$, Day $15(p=0.048)$, Day 16 $(p=0.021)$, Day 18-Day $24(p=0.012, p=0.029, p=0.022$, $p=0.0014, p=0.001, p=0.002$, and $p<0.001)$, and Day 48 $(p=0.004)$. In the 3 -d EtOH group, there were no significant difference from administration 1 (Day 3) until Day 24, but the locomotor sensitization was appeared on Day $30(p=0.009)$, Day $36(p=0.044)$, Day $42(p=0.001)$, and Day $48(p=0.006)$. In the 6-d EtOH group, locomotor activity was significantly different $v s$. administration 1 (Day 6) on Day $36(p<0.001$ ), Day $42(p<0.001)$, and Day $48(p=0.003)$.

After Day 48, we examined the locomotor sensitization after withdrawal from EtOH. Some mice died naturally during the experiment. One mouse each in the daily EtOH and 3-d EtOH groups died before Day 81 of the experiment. Between Days 81 and 186, 1 mouse died in the daily EtOH group. On Day 81, about 1 month after the last EtOH administration (Day 48), sensitization was observed in the daily EtOH $(p=0.009)$, 3-d EtOH $(p=0.002)$, 6-d EtOH $(p<0.001)$, and saline $(p=0.001)$ groups.

However, after a long period without EtOH exposure (Day 186), sensitization was markedly diminished, as evidenced by levels of locomotion near those measured following the first administration of the test substance in each group (daily EtOH group ( $p=0.889)$; 3-d EtOH group $(p=0.897)$; 6-d EtOH group $(p=0.786)$ and saline group $(p=1.000)$.

In Fig. 5C, we examined the main effects of treatment (saline, daily EtOH, EtOH every $3 \mathrm{~d}$, and EtOH every 6d) and number of administrations (1 to 8 times) using two-way ANOVA. EtOH-induced locomotion was significantly affected by treatment $[F(3,244)=237.7 ; p<0.001]$ and the number of EtOH administrations $[F(7,244)=59.7 ; p<0.001]$, with an interaction between dose and number of administrations $[F(21$, $244)=46.2 ; p=0.002]$. Using the Bonferroni's post-hoc test (vs. administration 1), significant sensitization was revealed in the daily EtOH group after administrations $6(p=0.043)$, $7(p<0.001)$, and $8(p=0.005)$, as well as in the 3 -d EtOH group after administration $6(p=0.025)$ and in the 6 -d EtOH group after administrations $6(p<0.001), 7(p<0.001)$, and 8 $(p=0.004)$, but not in the saline group. In the comparison of the 4 groups at administration 8 using Tukey's post-hoc test, the locomotor activity of the daily EtOH, 3-d EtOH, and 6-d EtOH groups $(2 \mathrm{~g} / \mathrm{kg} \mathrm{EtOH})$ were significantly higher than that of the saline group $(p<0.001, p=0.001$, and $p<0001$, respectively) with no significant difference among the daily EtOH, 3-d EtOH, and 6-d EtOH groups. Thus, the administration interval altered the rate of sensitization.

\section{DISCUSSION}

Dose and Time Dependence of EtOH Administration on CPP High EtOH doses (from 2 to $4 \mathrm{~g} / \mathrm{kg}$ ) are generally applied with agonists and antagonists in knockout mouse models 
to investigate the mechanisms of EtOH reward, because lower EtOH doses induce little or no CPP. ${ }^{8-11,29)}$ Thus, the rewarding effects of low-dose/long-term EtOH administration protocols, which may have clinical relevance by modeling the early phase of the progression to alcoholism, are unknown. To this end, we investigated the reward strength of low-dose EtOH using several dose-term regimens with a constant total dose. We found that moderate and long EtOH conditioning regimens induced CPP less effectively than higher doses for shorter periods, suggesting that moderate $\mathrm{EtOH}$ doses, even for an extended period, carry a lower risk of reward progression/sensitization than high EtOH doses over a shorter period.

However, lower EtOH doses can still be rewarding; the EtOH $(1,0.5 \mathrm{~g} / \mathrm{kg})$ groups showed CPP in comparison with that of their matched saline groups after 2 weeks (Figs. 1B, C). Nonetheless, low-dose/long-term EtOH administration conditions appeared less rewarding after 4 and 8 weeks. The repetitive conditioning/post-test would evoke the adaptation to the procedures in the subjects, since all CPP responses by EtOH $(0.5-1 \mathrm{~g} / \mathrm{kg})$ were observed only at 2 weeks (1st post test) but disappeared thereafter (4, 8 weeks). Unlike CPP induced by a moderate dose of $\mathrm{EtOH}$, the CPP values of the EtOH $(2 \mathrm{~g} / \mathrm{kg})$ after 2 weeks of conditioning were not significantly different from that of the group exposed to 4 weeks of conditioning (Fig. 3C). Collectively, these data suggest that lower $\mathrm{EtOH}$ doses but not higher $\mathrm{EtOH}$ dose are insufficient to induce the reproducible CPP responses, which could be detected for more than 2 weeks.

Corbit et al. reported that EtOH self-administration was less sensitive to devaluation (i.e., less flexible) after 8 weeks of drinking than after 1 week of drinking. ${ }^{30)}$ Behavioral inflexibility after 8 weeks of EtOH drinking was associated with a shift in the contribution of specific dorsal striatum subregions to behavioral control. Specifically, dorsomedial striatum (DMS) inactivation reduced EtOH-seeking after limited selfadministration, but was without effect after longer self-administration times, when dorsolateral striatum (DLS) inactivation reversed the insensitivity to devaluation. Although EtOH selfadministration and CPP deal with different parameters in drug dependence, low-dose EtOH regimens might be insufficient to induce a similar shift in behavioral control from DMS to DLS during extended conditioning in CPP.

Development of Sensitization and Place Preference to EtOH in the Black and White Chambers Illumination had a powerful influence on reward value, because even the highdose/short-term group did not exhibit CPP when EtOH was associated with a white chamber, despite the fact that the mice showed no baseline chamber preference. Moreover, locomotor sensitization was also mitigated in the white chamber (Experiment 3). Before EtOH administration, however, locomotor activity was higher in the dark, black chamber, consistent with the nocturnal nature of these animals. In contrast to illumination, another aspect of the context, floor texture, had no effect.

During the 300-s locomotion test, peak locomotor activity occurred 60-120s after EtOH administration (Figs. 3D, E). The EtOH-treated mice walked rapidly and appeared to stagger, especially in the later parts of the test. With repeated EtOH administrations, peak locomotion increased during the 60-120s epoch in both black and white chambers, whereas the subsequent decrease during the 240-300s epoch was smaller in the black, dark chamber, which may account for the greater sensitization under this condition. In accordance with enhanced sensitization, the CPP scores in the black chamber were higher than those in the white chamber. The CPP scores induced by a high dose of EtOH $(2 \mathrm{~g} / \mathrm{kg})$ did not decrease as the number of conditioning sessions increased.

Our results suggest that differences in brightness affected the reward value of EtOH during the $300 \mathrm{~s}$ conditioning period. Peak locomotor activity induced by EtOH was also observed within $300 \mathrm{~s}$. In the post-conditioning test after the 300 -s locomotion measurement, mice from the black and white chambers were immediately placed back into the same chamber.

We suggest that differences in anxiety caused by the dark and light conditions, as well as the level of locomotor activity in the CPP chambers, contributed to the effect of illumination on reward value. Cunningham and Zerizef reported CPP from visual and spatial cues in the light or in the dark. ${ }^{31)}$ Further, Varela et al. ${ }^{32)}$ reported that chronic complete light deprivation for 4 weeks inhibited appetitive associative learning induced by EtOH. Although methamphetamine and $\mathrm{EtOH}$ have very different mechanisms and subjective effects, a larger cage in which mice can move freely has been linked to greater methamphetamine-induced sensitization. ${ }^{19)}$ Therefore, the development of sensitization depends on the locomotor space used in the study and concomitant activity. The present results support the notion that context influences locomotor sensitization, because locomotor activity in the dark chamber was greater than that in the light chamber. Because rodents are nocturnally active, the drinking-in-the-dark (DID) model is used to examine binge-like ethanol consumption during periods of darkness. ${ }^{33-35)}$ EtOH conditioning in the dark chamber leads to arousal effects in mice and influences the rewarding effects of $\mathrm{EtOH}$, as seen in the present study.

Dose Dependence of EtOH-Induced Sensitization in Locomotion We also examined the influence of EtOH dose (Experiment 4) on EtOH-induced sensitization. On the first administration, EtOH-induced locomotion increased in a dosedependent manner (Fig. 4A). In addition, sensitization during repeated high-dose $\mathrm{EtOH}(2 \mathrm{~g} / \mathrm{kg})$ administration was stronger than that produced by repeated moderate-dose administration EtOH (1 g/kg) (Fig. 4B). Low-dose EtOH $(0.5 \mathrm{~g} / \mathrm{kg})$ did not induce locomotor sensitization.

Effect of EtOH Administration Interval on Sensitization Sensitization was also dependent on the conditioning interval, because the rate of locomotor enhancement differed among groups conditioned at different intervals (Experiment 5). Similarly, intermittent cocaine administration has been shown to induce stronger sensitization than continuous administration. ${ }^{20)}$ However, by the eighth EtOH administration, there were no significant group differences in the degree of locomotor sensitization (Fig. 5C). Thus, the dependence of EtOH locomotor sensitization on the conditioning interval appears to be weak.

EtOH sensitization was persistent in all EtOH-treated groups 1 month after withdrawal from EtOH (Fig. 5B). Boehm et al. reported that contextual learning affected $\mathrm{EtOH}-$ induced post-sensitization and showed that increased locomotor activity was maintained by EtOH administration every $14 \mathrm{~d}$ (for a period of $84 d) .{ }^{36)}$ The number of EtOH administrations was positively correlated with the number of days during which sensitization persisted. In the daily EtOH and saline groups, 
body weight increased after Day 48 . There were no significant differences in body weight among the 4 treatment groups (saline, daily EtOH, 3-d EtOH, and 6-d EtOH groups). The average of body weight of the 4 groups on Days 48, 84, and 186 was $28.3 \pm 0.4 \mathrm{~g}, 30.9 \pm 0.5 \mathrm{~g}$, and $33.2 \pm 0.7 \mathrm{~g}$, respectively, and these mice showed no withdrawal behavior. In all 4 groups, locomotor sensitization was diminished on Day 186 in comparison with that observed after the first EtOH administration. The reduction in EtOH locomotor sensitization over time may be a result of synaptic plasticity, ${ }^{37)}$ which could diminish the value of $\mathrm{EtOH}$ reward after a long period of discontinuation.

Effects of Acam on EtOH-Induced CPP To confirm the reward value of $\mathrm{EtOH}$ in our protocol, we investigated whether EtOH-induced CPP was blocked by Acam. Chester et al. reported that pretreatment with Acam $(400 \mathrm{mg} / \mathrm{kg}) 14$ and $2 \mathrm{~h}$ before administration of EtOH $(2 \mathrm{~g} / \mathrm{kg}$, i.p.) prevented the development of EtOH locomotor sensitization. ${ }^{38)}$ For the preclusive CPP protocol, Mcgeehan and Olive ${ }^{12)}$ pre-treated mice with Acam (i.p.) $10 \mathrm{~min}$ prior to ethanol administration during conditioning, whereas Kurokawa et al. ${ }^{13)}$ administered Acam (p.o.) $1 \mathrm{~h}$ before the post-test for the therapeutic CPP protocol. We performed the preclusive protocol by administering Acam (p.o.) before every conditioning trial (Experiment 2). As shown in Fig. 2, Acam $(600 \mathrm{mg} / \mathrm{kg}$; p.o.) significantly reduced the development of EtOH-induced CPP. These results confirm the value of our dosing protocol for further studies on Acam.

\section{CONCLUSION}

We examined the relationship between moderate $\mathrm{EtOH}$ intake and reward by assessing CPP and locomotor sensitization. The CPP scores produced by a long conditioning period to a moderate dose of EtOH were lower than those produced by a shorter EtOH conditioning regimen involving high EtOH doses. EtOH-induced CPP and locomotor sensitization were enhanced in the dark, black chamber, suggesting that paired associative learning affected the reward value of EtOH. EtOHinduced sensitization was markedly affected by the EtOH dose, but very little by the dosing interval. Moderate, but long-term, EtOH intake may confer a lower risk for reward progression and sensitization than heavy intake for shorter periods. Moreover, Acam preclusively inhibited the development of EtOH-induced CPP. These findings will support efforts aimed at developing strategies to prevent alcohol abuse and preclude the development of alcoholism.

Acknowledgment We thank Izumi Nomura, Frontier Laboratories of Value Creation, SAPPORO BREWERIES LTD., for help with the animal experiments.

Conflict of Interest Toshitaka Nabeshima serves as a consultant to SAPPORO BREWERIES LTD. Chikako Shimizu, Yasuhiro Oki, Yutaka Mitani, and Takeshi Nakamura are employees of SAPPORO BREWERIES LTD.

\section{REFERENCES}

1) Schmidt W, Popham RE. Heavy alcohol consumption and physical health problems: a review of the epidemiological evidence. Drug Alcohol Depend., 1, 27-50 (1975).

2) Bagnardi V, Blangiardo M, La Vecchia C, Corrao G. Alcohol consumption and the risk of cancer. a meta-analysis. Alcohol Res. Health, 25, 263-270 (2001).

3) World Health Organization. "Global strategy to reduce the harmful use of alcohol, Genova." WHO Library Cataloguing-in-Publication Data. 〈http://www.who.int/substance_abuse/msbalcstragegy.pdf.〉, cited (2010).

4) Osaki Y, Matsushita Y, Shirasaka T, Hiro H, Higuchi S. Nationwide survey of alcohol drinking and alcoholism among Japanese adults. Jpn. J. Alcohol Drug Depend., 40, 455-470 (2005).

5) Littleton J. Neurochemical mechanisms underlying alcohol withdrawal. Alcohol Health Res. World, 22, 13-24 (1998).

6) Clapp P, Bhave SV, Hoffman PL. How adaptation of the brain to alcohol leads to dependence: a pharmacological perspective. Alcohol Res. Health, 31, 310-339 (2008).

7) Gamsby JJ, Templeton EL, Bonvini LA, Wang W, Loros JJ, Dunlap JC, Green AI, Gulick D. The circadian Per1 and Per2 genes influence alcohol intake, reinforcement, and blood alcohol levels. Behav. Brain Res., 249, 15-21 (2013).

8) Cunningham CL, Prather LK. Conditioning trial duration affects ethanol-induced conditioned place preference in mice. Anim. Learn. Behav., 20, 187-194 (1992).

9) Cunningham CL, Gremel CM, Groblewski PA. Drug-induced conditioned place preference and aversion in mice. Nat. Protoc., 1, 1662-1670 (2006).

10) Voorhees CM, Cunningham CL. Involvement of the orexin/hypocretin system in ethanol conditioned place preference. Psychopharmacology, 214, 805-818 (2011).

11) Font L, Cunningham CL. Post-retrieval propranolol treatment does not modulate reconsolidation or extinction of ethanol-induced conditioned place preference. Pharmacol. Biochem. Behav., 101, 222-230 (2012)

12) McGeehan AJ, Olive MF. The anti-relapse compound acamprosate inhibits the development of a conditioned place preference to ethanol and cocaine but not morphine. Br. J. Pharmacol., 138, 9-12 (2003).

13) Kurokawa K, Mizuno K, Shibasaki M, Higashioka M, Oka M, Hirouchi M, Ohkuma S. Acamprosate suppresses ethanol-induced place preference in mice with ethanol physical dependence. J. Pharmacol. Sci., 122, 289-298 (2013).

14) Ishikawa K, Nitta A, Mizoguchi H, Mohri A, Murai R, Miyamoto Y, Noda Y, Kitaichi K, Yamada K, Nabeshima T. Effects of single and repeated administration of methamphetamine or morphine on neuroglycan $\mathrm{C}$ gene expression in the rat brain. Int. J. Neuropsychopharmacol., 9, 407-415 (2006).

15) Niwa M, Nitta A, Mizoguchi H, Ito Y, Noda Y, Nagai T, Nabeshima T. Novel molecule "shati" is involved in methamphetamine-induced hyperlocomotion, sensitization, and conditioned place preference. $J$. Neurosci., 27, 7604-7615 (2007).

16) Niwa M, Nitta A, Yamada Y, Nakajima A, Saito K, Seishima M, Shen L, Noda Y, Furukawa S, Nabeshima T. An inducer for glial cell line-derived neurotrophic factor and tumor necrosis factoralpha protects against methamphetamine-induced rewarding effects and sensitization. Biol. Psychiatry, 61, 890-901 (2007).

17) Steketee JD, Kalivas PW. Drug wanting: behavioral sensitization and relapse to drug-seeking behavior. Pharmacol. Rev., 63, 348-365 (2011).

18) Didone V, Masson S, Quoilin C, Seutin V, Quertemont E. Correlation between ethanol behavioral sensitization and midbrain dopamine neuron reactivity to ethanol. Addict. Biol., Dec. 24 (2014). 10.1111/adb.12216 [Epub ahead of print]

19) Hirabayashi M, Saito $T$, Tadokoro $S$. Differential sensitization to ambulation-increasing effect of methamphetamine after repeated administration to mice in activity cages of different sizes. Jpn. J. Pharmacol., 57, 91-97 (1991).

20) Hirabayashi M, Tadokoro S. Sensitization to ambulation-increasing effects of cocaine after repeated administration in mice-roles of 
dose and interval of administration as well as experimental environments. Arukoru Kenkyuto Yakubutsu Ison, 27, 91-102 (1992) (in Japanese).

21) Robinson TE, Berridge KC. The neural basis of drug craving: an incentive-sensitization theory of addiction. Brain Res. Brain Res. Rev., 18, 247-291 (1993).

22) Broadbent J, Kampmueller KM, Koonse SA. Role of dopamine in behavioral sensitization to ethanol in DBA/2J mice. Alcohol, 35, 137-148 (2005).

23) Crabbe JC, Harkness JH, Spence SE, Huang LC, Metten P. Intermittent availability of ethanol does not always lead to elevated drinking in mice. Alcohol Alcohol., 47, 509-517 (2012).

24) Horio M, Kohno M, Fujita Y, Ishima T, Inoue R, Mori H, Hashimoto K. Role of serine racemase in behavioral sensitization in mice after repeated administration of methamphetamine. PLoS ONE, 7, e35494 (2012).

25) Ide S, Minami M, Satoh M, Uhl GR, Sora I, Ikeda K. Buprenorphine antinociception is abolished, but naloxone-sensitive reward is retained, in mu-opioid receptor knockout mice. Neuropsychopharmacology, 29, 1656-1663 (2004).

26) Suzuki T, Kato H, Tsuda M, Suzuki H, Misawa M. Effects of the non-competitive NMDA receptor antagonist ifenprodil on the morphine-induced place preference in mice. Life Sci., 64, PL151-PL156 (1999).

27) Funada M, Sato M, Makino Y, Wada K. Evaluation of rewarding effect of toluene by the conditioned place preference procedure in mice. Brain Res. Brain Res. Protoc., 10, 47-54 (2002).

28) Cunningham CL, Ferree NK, Howard MA. Apparatus bias and place conditioning with ethanol in mice. Psychopharmacology, 170, 409-422 (2003).

29) Groblewski PA, Bax LS, Cunningham CL. Reference-dose place conditioning with ethanol in mice: empirical and theoretical analysis. Psychopharmacology, 201, 97-106 (2008).

30) Corbit LH, Nie H, Janak PH. Habitual alcohol seeking: time course and the contribution of subregions of the dorsal striatum. Biol. Psychiatry, 72, 389-395 (2012).

31) Cunningham CL, Zerizef CL. Effects of combining tactile with visual and spatial cues in conditioned place preference. Pharmacol. Biochem. Behav., 124, 443-450 (2014).

32) Varela P, Escosteguy-Neto JC, Coelho CT, Mello LE, da Silveira DX, Santos-Junior JG. Chronic light deprivation inhibits appetitive associative learning induced by ethanol and its respective c-Fos and pCREB expression. Int. J. Neuropsychopharmacol., 17, 1815-1830 (2014).

33) Nuutinen S, Vanhanen J, Mäki T, Panula P. Histamine h3 receptor: a novel therapeutic target in alcohol dependence? Front. Syst. Neurosci., 6, 36 (2012).

34) Sprow GM, Thiele TE. The neurobiology of binge-like ethanol drinking: evidence from rodent models. Physiol. Behav., 106, 325-331 (2012).

35) Carvajal F, Alcaraz-Iborra M, Lerma-Cabrera JM, Valor LM, de la Fuente L, Sanchez-Amate MD, Cubero I. Orexin receptor 1 signaling contributes to ethanol binge-like drinking: Pharmacological and molecular evidence. Behav. Brain Res., 287, 230-237 (2015).

36) Boehm SL 2nd, Goldfarb KJ, Serio KM, Moore EM, Linsenbardt DN. Does context influence the duration of locomotor sensitization to ethanol in female DBA/2J mice? Psychopharmacology, 197, 191-201 (2008)

37) Abrahao KP, Ariwodola OJ, Butler TR, Rau AR, Skelly MJ, Carter E, Alexander NP, McCool BA, Souza-Formigoni ML, Weiner JL. Locomotor sensitization to ethanol impairs NMDA receptor-dependent synaptic plasticity in the nucleus accumbens and increases ethanol self-administration. J. Neurosci., 33, 4834-4842 (2013).

38) Chester JA, Grahame NJ, Li TK, Lumeng L, Froehlich JC. Effects of acamprosate on sensitization to the locomotor-stimulant effects of alcohol in mice selectively bred for high and low alcohol preference. Behav. Pharmacol., 12, 535-543 (2001). 